\title{
Silent Negotiations: A Case Study of Roles and Functions Utilized by Students, Teachers, and Mentors in Project-based, Telementoring Relationships
}

\author{
Kallen Tsikalas and Katherine McMillan-Culp \\ Center for Children \& Technology, EDC • 96 Morton Street $\bullet$ New York, NY 10014 \\ Phone: (212) 807.4200; Fax: (212) 633.8804 \\ E-mail: ktsikalas@edc.org; kmcmillan@edc.org
}

\begin{abstract}
This paper describes novel research that we conducted as part of the Portals project (NSF Grant \#CDA-9616990). We examined the roles, functions and strategies that students, teachers, and mentors bring to complex on-line, project-based learning experiences. Data for our study consisted of site observations, interviews, student work samples and videotaped project presentations from twelve project-based mentoring relationships that were conducted entirely or partially on-line. We analyzed these data within the frameworks of mentoring, cognitive apprenticeship, and educational network interactions. Additionally, we introduced a web-based tool (Portals) to facilitate more effective communication in such mentoring situations and documented how and when it was used. We learned that mentors often take their cues from students about how to proceed in mentoring relationships and that they may not always be aware of the roles they play; and that teachers are important co-mentors in the process, particularly in creating cultures of collaboration in their classes and explicitly attending to and supporting students' communication skills. Technology was also an important player in these relationships. Recommendations for improving telementoring designs are included in the paper.
\end{abstract}

Keywords: Mentoring, Learning environments, Computer-mediated communication.

\section{Introduction}

It is the end of another school year, and Linda Albey--an enthusiastic teacher of eight years--reflects on the successes and challenges of her project-based computational science class. The 20 tenth, eleventh, and twelfth grade students in Linda's rural Tennessee class have just completed projects on subjects that range from modeling the flight of a condor to predicting the probability of power-lines being struck by lightning to determining whether antimatter galaxies might exist.

Most of these student teams have worked with on-line mentors to define problems, develop research plans, create mathematical and computational models of systems, and interpret and communicate the results of their studies. The mentoring relationships have been both successful and challenging, and Linda comments:

I'm starting to see as I have more projects behind me, that some of these mentors want to have this on-going kind of conversation with the students and work with them to develop their project. Others want to have a plan in mind, teach the kids what to do, then out. I don't know that one's better. We're probably going to have to accommodate both styles...

Linda and her students represent a growing trend in Internet-enhanced education of engaging adults from outside the classroom as experts, advisors or consultants in children's learning experiences. This phenomenon is cause for both excitement and reflection; and over the last few years, groups of researchers have begun to examine telementoring relationships more systematically. Most notable among these researchers are O'Neill (1998); Bennett, Hupert, Tsikalas, Meade \& Honey (1998); and Harris (1995). Their research is rooted in seminal studies of mentoring in education (primarily undergraduate and professional), management, and psychology.

We sought to further grow this body of knowledge by characterizing the conditions and structures of learning environments (physical and virtual) that contribute to satisfying and effective project-based, telementoring 
relationships. Our work extends the present corpus particularly by identifying and describing students' and teachers' roles and strategies in such telementoring experiences.

As part of the Portals project, we examined twelve project-based mentoring relationships that were conducted entirely or partially on-line. Portals was a research and development project funded by the National Science Foundation (NSF Grant \#CDA-9616990). The mentoring relationships that we examined involved 40 high school students, 5 teachers, and 12 mentors. Classes were located in Iowa and Tennessee and were part of the Adventures in Supercomputing (AiS) program, which is supported by the Department of Energy. At the time of the Portals project, CCT had worked with this program for three years, conducting evaluations of student learning (Honey, McMillan, Tsikalas, \& Grimaldi, 1994; Honey, McMillan, Tsikalas, \& Light, 1995).

Data for our study consisted of mid- and post-project site observations and interviews with teachers and students, post-project interviews with mentors, student work samples and videotaped project presentations. Using a three-stage analysis process, we identified the roles and functions employed by teachers, mentors and students to develop effective relationships. We analyzed these within the frameworks of mentoring (Bennett et al., 1998; Jacobi, 1991; O'Neill, 1998; NAS, 1997; Sullivan, 1994), cognitive apprenticeship (Collins, Brown, \& Newman, 1989; Scardamalia, Bereiter, \& Steinbach, 1984), and educational network interactions (Levin, 1995). Additionally, we introduced a web-based tool (Portals) to facilitate more effective communication in such mentoring situations and documented how and when it was used. This paper will report our primary findings regarding the roles and functions demonstrated by students, mentors, and teachers in sustaining successful on-line mentoring relationships.

\section{Finding 1: Interpersonal and Learning Dynamics of Mentoring}

Mentoring is a notoriously fluid and idiosyncratic activity (Sullivan, 1992; Welch 1996) and not easy to define scientifically. This is due to its inherently social nature, the varied roles that mentors may play, the developmental level of mentees, and the variety of forms these relationships may assume (e.g., short-term vs. longterm, formal vs. natural, etc.). Jacobi (1991) reports a number of problems associated with the absence of a widely accepted operational definition of mentoring. O'Neill (1998) suggests that this diversity in types of assistance and support provided may itself be the defining characteristic of mentoring.

We adopted the language of the National Academy of Science and defined successful mentoring as the development of relationships that "advance the educational and personal growth of the student (NAS, 1997)." This broad definition--which encompasses psychosocial, career, and academic functions (Jacobi, 1991)--best reflected the goals of the teachers in our study. These teachers indicated that they hoped telementoring experiences would provide their students with:

- access to scientific content expertise that extended beyond their own;

- experience with how real scientists make choices about research questions, methods, data presentation, and data interpretation;

- exposure to a variety of scientific careers and the personal side of science; and

- opportunities to learn to communicate with adults in the real world who weren't their parents or teachers.

Because project-based mentoring relationships are co-constructed by students, mentors and teachers, it is often difficult to disentangle the functions and strategies that lead to successes or challenges. However, in the following stories, we have described strategies used or choices made by a group of students (in the first) and a mentor (in the second) that complicated the creation of constructive working relationships.

\section{Case \#1}

Chloe, James and Madison, tenth grade students in a small Iowa town, researched the mechanics of the levitron for their project. The levitron is a small device that resembles a spinning top and pedestal. When rigged correctly (with wedges and magnets) and spun, the top appears to defy gravity by hovering above the pedestal. Chloe received this "toy" for Christmas and brought it into class for her teammates to inspect. Soon thereafter, it became the basis of their project. The team's mentor, found through an Internet search, was a spirited professor of electrical engineering at an East Coast university and devotee of the levitron. Professor Johnson was pleased to 
work with the students, indicating that he was impressed they would even consider tackling a subject as complex as this one. He regularly praised the team for their effort.

During the project, Johnson provided information and equations explaining the device. He also encouraged the students to experiment, proposing different conditions they might investigate and posing questions about what they discovered. After protracted silences in which they did not respond to any of his questioning, the students finally told their mentor that they were unable to get the levitron to levitate. Johnson recalled, "For the longest time, they were unable to get the thing [levitron] to work... When she [Chloe] finally admitted this to me, I arranged to have a very instructive videotape sent to them."

The students also never revealed to their mentor the extent to which they did not understand the formulas he had supplied. They described the equations as monsters and indicated that they learned how to code the operations but did not really know what the numbers meant. For instance, the team calculated the most stable point for levitation--a value with $\mathrm{x}, \mathrm{y}$, and $\mathrm{z}$ coordinates--but they didn't know if this point actually existed in space. James explained, "They're just numbers. It's not the distance up here; it's just where the systems are solved. I don't really know if that's an actual area."

Enacting a strategy of concealment--hiding information in order to preserve an image of success--Chloe commented, "We were embarrassed to admit our weaknesses to him, and we didn't want him to feel like he had to take hours off his work and explain this to us in real simple terms." James added: "I think we didn't want to let him down."

Professor Johnson fully expected the students to have questions and need assistance, but he was unprepared for their inability or unwillingness to effectively represent what they didn't understand. Nor did he anticipate a culture in which pleasing the adult could take precedence over learning:

What they [the students] should be encouraged to do, is to formulate their questions, formulate their concerns, and be quite willing to be honest and say, "We're lost" or "This is boring, and please explain why we have to do it." You know, they should be able to be a little bit more honest about what it is that is holding them up or why things aren't going they way they might want to go. Now, in direct contact, those kinds of messages are much more easily conveyed. But by email, it's different... It's extremely difficult.

Our research indicated that mentors often took their cues from students in these relationships--a finding which supports O'Neill's (1998) contention that students in telementoring relationships are often in positions of greater control than mentees in typical mentoring relationships. We found that this shift of circumstance (wherein young people have relatively greater power in and responsibility for developing their telementoring relationships) created complications when the students were not skilled in representing both what they knew and didn't know. Students' ability to effectively communicate these things is particularly critical in cases of on-line project mentoring in which mentors are unable to detect when and why students might be having trouble.

\section{Case \#2}

Sophie and Adam were very self-directed and communicative students. They had a good deal of experience working on projects as well as background in upper-level math when they began their project on pharmacokinetic modeling. In fact, both had won awards for their projects in previous years-a fact they did not neglect to tell their mentor, Dr. James Hodgkiss. Working with Hodgkiss, a computational physicist, the students created a computer model of second hand smoke (the nicotine in environmental smoke) as it was metabolized by the body. Adam explained that they divided the body into six compartments - "vessel poor, vessel rich, liver, fat, kidney, and lung blood" - and then, controlling for several factors such as body mass, they calculated the percentage of nicotine metabolized in each of these compartments.

Dr. Hodgkiss was proud of the students and interested in the model for its own sake. He commented that he had "a bit of personal stake" in the project and hoped to further develop this line of work in his own research. He treated Sophie and Adam like colleagues and even planned to publish a paper on their project, naming them as co- 
authors. However, occasionally Hodgkiss did not explicitly involve the students in important decisions about their work. For instance, he repeatedly changed his mind about which code (model) they should use. Adam explained, "Our mentor was like, 'I don't like this code; let's try another one.' As soon as we got that done, he'd say, 'I don't like this code, let's try another one." The students' teacher agreed that this revision process was a bit excessive and enigmatic, saying:

I think their mentor is pretty well on the ball; he helped them decide a lot of stuff. But the thing he did that kept them off guard, is they would almost have something ready, they thought, 'We've got it now.' And he'd come back and say, 'We've got to change this, I don't like that. We're going to throw it all away and start over here with this... And that frustrated the kids.

In interviews with our research team, Hodgkiss acknowledged that he had changed his position several times. As he saw how the students were working with the first model, he thought a different approach might be simpler: "I was thinking from a conceptual point of view, this would be easier to understand." Later, when he saw that the older model was "fitting the data better" and that it might also be "a little easier for them to explain," he suggested that they switch back to the earlier approach. In retrospect, the mentor noted that he should have better explained the differences between these models and why he had recommended changing from one to another.

In the opening passage of this paper, teacher Linda Albey reflected on two mentoring roles/functions she had observed in her class. The mentoring literature supports her observations. Additionally, it indicates that mentors may be advisors, coaches, role models, friends, parents, tutors, skills consultants, career counselors, and critics among others (Ganser, 1994; NAS, 1997; Sullivan, 1994). Jacobi (1991) further specifies a number of mentoring functions.

Our research suggested that mentors were rarely fully aware of the panoply of roles/functions they might employ. Particularly in on-line relationships, mentors sometimes forget the importance of making their own thinking transparent, so that students may acquire not only domain knowledge but also heuristic and control strategies, such as how to make decisions about how to proceed in a task (Collins, 1989).

\section{Implications for the Design of Telementoring Experiences}

The mentoring literature emphasizes the importance of mentor training and preparedness in successful relationships (Bennett, 1998; Harris, 1995; NAS, 1997; Sullivan, 1994). However, these two cases of project-based, telementoring relationships as well as others we examined suggest that students need to be better prepared for mentoring relationships as well. Such preparation might include:

- Assuring students that while it may seem counter to what they've experienced in their schooling, being open and honest with their mentors about what they do and do not understand as well as what does and does not interest them is essential for a successful relationship;

- Providing opportunities for students to practice describing (in text) what they do and do not understand about a problem as well as when they're losing steam;

- Providing opportunities for students to receive feedback (e.g., through peer or teacher critiques) about these communicative acts; and

- Educating students about the various roles/functions mentors may exercise in helping them with their projects.

Additionally, our research suggested that student teams that were able to communicate and collaborate well with each other were also able to do so more effectively with telementors. While this finding may simply indicate that good communicators are good communicators regardless of their "audience," it may also imply that the team relationship could serve as a microcosm for the telementoring relationship. Hence, helping students to develop strong teams could improve their potential for success with on-line mentors.

Our studies also indicated that telementors may need to be made better aware of their multiple roles/functions in these relationships and, particularly, of the importance of making their thinking explicit. This type of information and feedback might best delivered by teachers at regular moments in the mentoring relationship. Jacobi's (1991) catalog of mentoring functions may be helpful. Among the functions it includes are: Providing 
acceptance, support, or encouragement; Offering advice or guidance; Helping mentees to bypass bureaucracy or gaining access to resources; Challenging mentees providing access to new opportunities or "plum assignments;" Helping mentees to clarify their values or goals; Coaching; Providing Information; Role modeling; Socializing mentees into particular cultures; Sponsoring or advocating for mentees; Stimulating mentees to acquire new knowledge; and Training or instructing

\section{Finding 2: Teachers as Co-mentors}

Past research has stressed the importance of facilitation in telementoring relationships (Bennett et al., 1998; Harris, 1995). This includes both human and structural mediation. For instance, O'Neill (1998, p. 47) asserts that "telementoring relationships are strongly influenced by the rituals and events of the classroom." Furthermore, he posits that the second most important element in the design of project-based telementoring is the activity structure that teachers craft. He defines this activity structure as a "set of roles and responsibilities for students, telementors, and the teacher, connected to a schedule of deliverables (1998, p. 54)."

Our research found evidence that supports these positions, but it also suggested the importance of human mediation. Consequently, we chose to further explore the roles that teachers assumed and the strategies they employed in facilitating their students' telementoring relationships. We used Levin's (1995) taxonomy of educational network interactions to frame this analysis. Levin's scheme identifies five general characteristics of such on-line educational activities: structure; process; mediation; community building; and institutional support.

In our study of five teachers and twelve student-mentor pairs, we observed seven general strategies/functions of teachers. Though most of these teacher strategies fell into the areas of mediation and community building, we found that two strategies in particular seemed highly related to successful mentoring relationships:

- Creating a classroom culture of collaboration among students and between students and many external resources. (Community building strategy)

- Explicitly attending to students' communication skills and needs by rehearsing important conversations and by providing opportunities for students themselves to teach or mentor. (Mediation strategy)

The following case illustrates these strategies in action.

\section{Case \#3}

Kyle Perkins has taught programming for the last ten years and project-based computational science for the last five. During our study, Mr. Perkins taught five different AiS classes and supervised 28 project groups, most with mentors. Perhaps because of the quantity of students and projects he was responsible for, he consistently encouraged young people to use each other as resources and to enlist the help of as many external people as possible. His was one of the few classes in which it was common for project groups to have several mentors and to work with these different experts simultaneously or sequentially depending on their needs. The practice of using multiple mentors is encouraged by the National Academy of Sciences (1997) and Association for Supervision and Curriculum Development (1994).

Students in Perkins' class also were assigned to manage hardware and software. They were treated as experts and were expected to help each other. Consequently, these students seemed to go first to other students when they had questions or needed help. They also shared resources. For example, the Hurricane project group obtained their mentor from another AiS team. The latter group was studying tornadoes but happened to discover a hurricane forecaster at the National Hurricane Center while participating in an on-line Q\&A bulletin board. They shared this information with the hurricane team who soon thereafter "shanghaied" him (in Perkins' words) but continued to consult him occasionally as their project required. There were no firm boundaries demarcating which mentor belonged to whom--indicative of the culture of sharing that seemed to have been established in Mr. Perkins' classes.

Perkins also placed great emphasis on students' communicating in as many ways as possible with their mentors, and he developed practical ways to support these exchanges. For example, he provided one mentor with an 
account on the school's server so that he (the mentor) could easily view students' computer code while simultaneously chatting with them. Similarly, he encouraged students to use multiple representations, most notably graphs and illustrations, when communicating with their mentors. About communication, Perkins remarked, "We know we need to be more aggressive. We can't just sit back and wait for them to ask us questions. We have to tell them what we know and tell them how we found out."

\section{Implications for the Design of Telementoring Experiences}

Our study suggested that in successful mentoring situations, teachers actually became co-mentors. For example, they provided students with opportunities to themselves be mentors--an important function of the mentor as advisor. Similarly, they helped students develop effective communication skills--a primary function of the mentor as skills consultant. Coaching students on "how to construct good questions and summaries... and critiquing their efforts" (Collins, pg. 461) is also an essential characteristic of a cognitive mentor.

Though they often assumed the role of co-mentors, teachers were seldom recognized as such by students or their mentors. This phenomenon has also been described in Lenert and Harris's (1994) analysis of conceptions of expertise in project-based telementoring relationships. These authors suggest that the nature of expertise in these situations must be revised to include both content knowledge and the skill and knowledge needed to facilitate learning.

We believe that it is always important for teachers to be active as co-mentors in the mediation of studentmentor relationships and also to be acknowledged by external mentors as having this role. Most of the mentors in our study were not cognizant of the types of support teachers were providing to students in this project-based setting. We recommend that in framing the experience for telementors (or in helping their students do so), teachers explicitly articulate their own responsibilities and activities in facilitating learning in this endeavor. They might do this by posting an activity structure, as O'Neill (1998) has suggested is important, or by sharing occasional progress reports with mentors. We recommend that periodic teacher-mentor communication be included and planned for as an integral part of the mentoring relationship.

\section{Finding 3: Technology and its Role in Mediating Communication}

We introduced Portals, a suite of web-based display and annotation tools designed to improve studentmentor conversations, into this environment. At the time, we were aware of the general mentoring climate in AiS but unaware of the range of teacher, student and mentor roles/functions, strategies and interactions that we learned through this research.

Portals is based on four key constructivist principles (Collins, 1989; Pea, 1994; Pea, Edelson, \& Gomez 1994):

1. Collective learning requires mutual points of reference--documents and/or artifacts shared among students, mentors, and teachers;

2. Effective learning conversations start where the students are; therefore the work and thoughts of students, rather than those of mentors, must be at the center of such conversations.

3. Reformulation, attempting to express problems and thoughts in multiple ways, is a productive way for students to enhance and communicate their own understandings.

4. Intelligence does not reside in tools, but rather in the people who use them and in the relationships they support.

The Portals application enables students to post and archive sets of "process documents" on a password-protected web-site. While the students' mentor, teacher, teammates and designated guests can access and view this site, only mentors may annotate the documents.

Process documents, in contrast to final products, are representations of students' thinking that are not expected to be fully developed or refined. Though similar to what O'Neill (1998) identifies as interim deliverables in his activity structure, process documentation in Portals was intended not only to provide mentors with regular progress reports but also to give them real windows into the ways students were conceptualizing and understanding their research endeavors. Process documents were intended to emphasize the formative nature of knowledge and to sanction young people to share their ideas and work as they went along--while their knowledge was still 
"imperfect." A Portals website and guide offered students and teachers suggestions for creating various types of process documents (including text summaries, concept maps and flow charts, pieces of programming code, illustrations and mental movies) and indicated points in the project development process at which certain documents might be most helpful.

While Portals was not widely used in the telementoring relationships we investigated (only three of the twelve project teams made use of the environment), we did learn from its implementation. Just as we learned that teachers must be included explicitly in student-mentor relationships, so too did we learn that teachers must be included prominently in technology designs and processes. We also observed that the assumptions inherent in technologies do not always match the predominant cultures of classrooms and mentoring relationships.

In particular, we learned that privileging the role of students as producers of knowledge and focusing on intermediate rather than final products of the thinking process, did not always fit with classroom culture, project requirements, or mentors' visions of their roles and functions. Additionally, we observed that students often did not know what kind of information to share with their mentors without substantial guidance from their teachers. For instance, one group of students studying eagle repopulation created a Portals process portfolio for their mentor that consisted almost entirely of photos of flying eagles--photos which provided their mentor with no information about what they were thinking or how they were proceeding with the project. Similarly, we learned that mentors did not always know how to consider representations of students' thinking that were not text-based. For instance, a group of students studying antimatter posted a "mental movie" illustrating how they imagined gamma radiation from matter-anti-matter collisions might travel through and be impeded by matter galaxies. Their mentor responded easily to the students' text questions about the matter but didn't quite know what to do with the illustration.

\section{Conclusions}

In the Portals research, we examined the roles/functions and strategies that students, teachers, mentors and technologies bring to complex on-line, project-based learning experiences. We learned that mentors often take their cues from students about how to proceed and that they may not always be aware of the roles they play; and that teachers are important co-mentors in the process, particularly in creating cultures of collaboration in their classes and explicitly attending to and supporting students' communication skills. To improve future telementoring designs, we recommend that:

- Students be better prepared for project-based telementoring experiences;

- Mentors be better informed about their potential roles/functions in these relationships, particularly the importance of making their own thinking visible in the on-line environment; and

- Teachers honor and explicitly state their own responsibilities and activities in facilitating students' learning in such endeavors.

\section{References}

Bennett, D., Hupert, N., Tsikalas, K., Meade, T., and Honey, M. (1998). Critical Issues in the Design and Implementation of Telementoring Environments. New York, NY: Center for Children and Technology, EDC. Also http://www.edc.org/CCT/cctweb/public/.

Collins, A., Brown, J.S., and Newman, S.E. (1989). Cognitive apprenticeship: Teaching the crafts of reading, writing, and mathematics. In Knowing, Learning and Instruction: Essays in Honor of Robert Glaser (L.B. Resnick, ed.). Hillsdale, NJ: Erlbaum.

Ganser, T. (1994). Metaphors for Mentoring: An Exploratory Study. Paper presented at the Annual Meeting of the American Educational Research Association, New Orleans.

Honey, M., McMillan, K., Tsikalas, K., and Grimaldi, C. (1994). Adventures in Supercomputing 1993-94 Evaluation: Final Report. New York, NY: Center for Children and Technology, EDC. Also http://www.edc.org/CCT/cctweb/public.

Honey, M., McMillan, K., Tsikalas, K., and Light, D. (1995). Adventures in Supercomputing 1994-95 Evaluation: Final Report. New York, NY: Center for Children and Technology, EDC. Also http://www.edc.org/CCT/cctweb/public.

Harris, J. (1995). Organizing and facilitating telecollaborative projects. The Computing Teacher, 22(5), 66-69.

Jacobi, M. (1991). Mentoring and undergraduate academic success: A literature review. Review of Educational Research, 61 (4), 505-532. 
Kerka, S. (1998). New Perspectives on Mentoring. ERIC Digest No. 194. Columbus, OH: ERIC Clearinghouse.

Lenert, K.F., \& Harris, J.B. (1994). Redefining Expertise and Reallocating Roles in Text-Based Asynchronous Teaching/Learning Environments. Machine-Mediated Learning, 4 (3).

Levin, J. (1995). Organizing educational network interactions: Steps towards a theory of network-based learning environments. Paper presented at the Annual Meeting of the American Educational Research Association, San Francisco.

National Academy of Sciences (1997). Adviser, Teacher, Role Model, Friend: On Being a Mentor to Students in Science and Engineering. Washington, DC: National Academy Press. Also http://www.nap.edu/readingroom/books/mentor.

O'Neill, D.K. (1998). Engaging Science Practice Through Science Practitioners: Design Experiments in K-12 Telementoring. (Doctoral Dissertation, Northwestern University, 1998.)

Pea, R. (1994). Seeing what we build together: Distributed multimedia learning environments for transformative communications. The Journal of the Learning Sciences, 3(3), 285-299.

Pea, R., Edelson, D., and Gomez, L. (1994). The CoVis Collaboratory: High school science learning supported by a broadband educational network with scientific visualization, videoconferencing, and collaborative computing. Paper presented at the Annual Meeting of the American Educational Research Association, San Francisco.

Scardamalia, M., C. Bereiter, and R. Steinbach (1984). Teachability of reflective processes in writing composition. Cognitive Science, 8, 173-190.

Sullivan, C.G. (1994). How to Mentor in the Midst of Change. Alexandria, VA: ASCD.

Welch, O.M. (1996). An Examination of Effective Mentoring Models in the Academy. Paper presented at the Annual Meeting of the American Educational Research Association, New York. 